\title{
Improving the Statement of the Corrective Security-Constrained Optimal Power-Flow Problem
}

\author{
Florin Capitanescu and Louis Wehenkel, Member, IEEE
}

\begin{abstract}
This letter proposes a formulation of the corrective security-constrained optimal power-flow problem imposing, in addition to the classical post-contingency constraints, existence and viability constraints on the short-term equilibrium reached just after contingency. The rationale for doing so is discussed and supported by two examples.
\end{abstract}

Index Terms-Optimal power flow, security-constrained optimal power flow.

\section{INTRODUCTION}

$\mathbf{T}$ HE security-constrained optimal power flow (SCOPF) problem has been formulated under two modes: "preventive" [1] and "corrective" [2], called PSCOPF and CSCOPF.

The CSCOPF computes an optimal combination of preventive and open-loop corrective controls. An implicit assumption of the classical CSCOPF formulation is that the system reaches a stable short-term equilibrium just after the contingency application and that it will survive until/during the post-contingency control actions are taken. However, this assumption is not always true, especially for stressed operating states and/or if voltage instability is a concern. Actually, since the CSCOPF naturally leads to more stressed operating conditions, it may significantly increase the risk of dynamic instabilities such as voltage collapse or cascading line trippings which could occur before the corrective control actions can be applied.

To mitigate this problem, we propose in Section II an improved CSCOPF formulation imposing existence and viability constraints on the short-term equilibrium reached just after contingency occurrence and before corrective controls are applied. In Section III, we provide examples where the classical CSCOPF formulation leads respectively to power-flow divergence just after contingency occurrence, or to severe overcurrents which could lead to cascading line trippings before any post-contingency control could be applied.

\section{IMPROVED STATEMENT OF THE CORRECTIVE SECURITY-CONSTRAINED OPTIMAL POWER FlOW}

The proposed improved approach to the CSCOPF problem can be compactly stated as follows:

$$
\begin{array}{cc}
\min f_{0}\left(\mathbf{x}_{0}, \mathbf{u}_{0}\right) & \\
\text { s.t. } \mathrm{g}_{k}\left(\mathbf{x}_{k}, \mathbf{u}_{k}\right)=\mathbf{0} & k=0, \ldots, c \\
\mathbf{g}_{k}^{0}\left(\mathbf{x}_{k}^{0}, \mathbf{u}_{0}\right)=\mathbf{0} & k=1, \ldots, c
\end{array}
$$

Manuscript received October 5, 2006. Paper no. PESL-00079-2006.

The authors are with the Department of Electrical Engineering and Computer Science, University of Liège, Belgium (e-mail: capitane@montefiore.ulg.ac.be; 1.wehenkel@ulg.ac.be).

Digital Object Identifier 10.1109/TPWRS.2007.894850

$$
\begin{aligned}
\mathbf{h}_{k}\left(\mathbf{x}_{k}, \mathbf{u}_{k}\right) & \leq \mathbf{h}_{k}^{\max } & k & =0, \ldots, c \\
\mathbf{h}_{k}^{0}\left(\mathbf{x}_{k}^{0}, \mathbf{u}_{0}\right) & \leq p_{k} \mathbf{h}_{k}^{\max } & k & =1, \ldots, c \\
\left|\mathbf{u}_{k}-\mathbf{u}_{0}\right| & \leq \Delta \mathbf{u}_{k}^{\max } & k & =1, \ldots, c
\end{aligned}
$$

where $f_{0}$ models the cost of preventive control actions, and, for the $k$ th system configuration ( $k=0$ corresponds to the pre-contingency configuration, while $k=1, \ldots, c$ correspond to the $c$ post-contingency configurations), $\mathbf{x}_{k}$ is the vector of state variables, $\mathbf{u}_{k}$ is the vector of preventive and corrective control variables. Equality constraints (2) and inequality constraints (4) impose the feasibility of the pre-contingency and corrected post-contingency states. On the other hand, equality constraints (3) and inequality constraints (5) impose, for each contingency, the existence and viability of the intermediate state reached just after contingency occurrence and before application of corrective control actions.

Equality constraints (2) and (3) are essentially the AC bus power balance equations, while the inequality constraints (4) and (5) concern physical limits of equipments (e.g., bounds on: generators active/reactive powers, transformers equipped with tap-changer ratio, shunts reactance, phase shifters angle, etc.) and operational limits (e.g., on branch currents and voltage magnitudes). Note that $p_{k} \geq 1$ is a scalar value modeling how much the constraints just after the contingency application are relaxed with respect to the permanent limits. Inequalities (6) are "coupling" constraints between the base case and post-contingency values of control variables aimed at preventing unrealistic values of corrective control variables; $\Delta \mathbf{u}_{k}^{\max }$ is the vector of maximal allowed variations of control variables between the base case and $k$ th post-contingency state.

With respect to the classical CSCOPF formulation, we thus propose to include the additional constraints (3) which ensure, for each contingency, the existence of a post-contingency equilibrium point when the control variables are frozen to their pre-contingency values, and the constraints (5), which ensure that in this state the operational limits are not violated too much, so that the corrective control action $\mathbf{u}_{k}-\mathbf{u}_{0}$ may be taken before cascading tripping or equipment damage occurs. Note that the exact nature of these additional constraints will depend on the system dynamics and degree of safety one wants to ensure. For example, by using a constant power load model to formulate (3) and $p_{k}=1$ in (5), the solution of our modified CSCOPF becomes actually as conservative and costly as that of a PSCOPF.

\section{NUMERICAL ReSUlTS}

We present two examples supporting the interest of our formulation on a 60-bus/23-generator test system, which is a modified variant of the "Nordic32" system [3]. We consider, for sim- 
plicity, a single contingency (the loss of a major branch). In our simulations we use the interior-point method to solve optimization problems (see [44]).

We solve the CSCOPF problem of minimizing the overall generation cost with and without the additional constraints and compare their results, for two different scenarios. In both cases, the control variables are the active/reactive powers of all generators. In these simulations, we consider that all equality constraints are the AC bus active/reactive power balance equations with constant power loads, and inequality constraints are bounds on generator active/reactive powers and current limits for the 81 branches. In the coupling constraints we assume for every generator that $\Delta \mathbf{u}_{k}^{\max }$ is $2 \%$ of its active power physical range of variation.

In the first example we choose a highly loaded base case. We run the CSCOPF without the additional constraints and, at the optimal solution, we observe that $302 \mathrm{MW}$ are shifted in corrective mode between several generators, to ensure post-contingency state feasibility. Note that most of the coupling constraints are binding at the optimum, indicating that these generators have exhausted all their available corrective control capacity, while none of the inequality constraints (4) are binding. However, when simulating the contingency at the so-optimized base case, by a classical AC power-flow software without applying the corrective controls, the computation diverges. Actually, we have determined that the minimum load shedding to restore post-contingency feasibility leads to the curtailment of $49 \mathrm{MW}$ and 32 Mvar at two load buses. This means that the preventive/corrective control combination computed with the classical CSCOPF assumptions, leads to a situation of high risk of voltage collapse. Note that, as expected, we have found in our experiments many such cases, admittedly especially among (very) stressed operating points.

When using the additional constraints in the CSCOPF formulation we find that none of the inequality constraints (4) or (5), for $p \geq 1$, are binding at the optimum. Since the set of power-flow equations just after contingency (3) is obviously more constraining than the set relative to the corrected state (2), and that constraints (3) are included also in the classical PSCOPF, the optimal solution of our CSCOPF approach actually coincides here with that of the classical PSCOPF. This suggests that contingencies which do not have an equilibrium point just after the contingency should be treated in preventive mode without relying on corrective control.

These conclusions hold true at least as long as one uses a constant (apparent) power model for loads in pre- and any post-contingency state, a common assumption in SCOPF computations. This implicitly assumes that load is restored before the corrective actions (impacting load restoration) start. Clearly, the use of a different short-term load model, for the power-flow equations just after contingency (e.g., ZIP, depending exponential of voltage, etc.), deserves further investigation.

We now choose a thermal "congested" operating point, for which at the OPF solution (CSCOPF model without contingency constraints) one branch current constraint is binding. We then run the classical CSCOPF and find that one (different) branch current is at its maximum value in the post-contingency

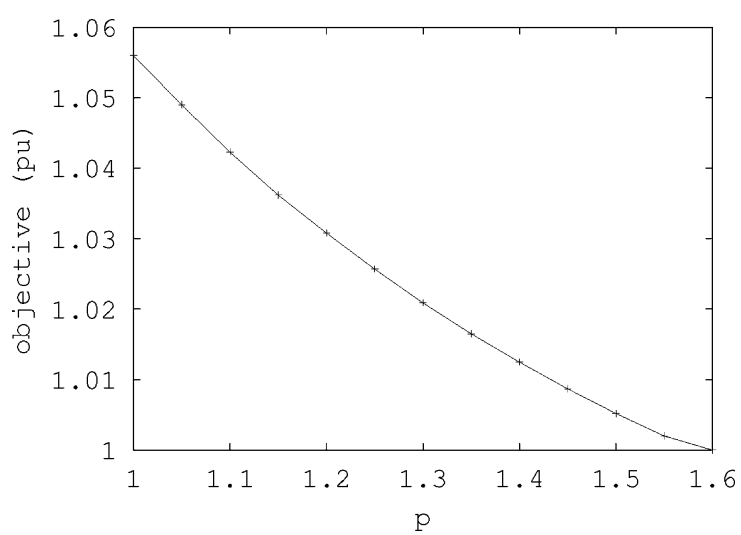

Fig. 1. Influence of the relaxation factor $p$ on the objective value.

state. Now, by simulating the contingency at the classical CSCOPF optimal base case, several branches are overloaded among $124 \%$ and $158 \%$. These extremely high overloads correspond to the system state right after contingency, while the optimal corrective actions proposed by the classical CSCOPF approach succeed to remove all these overloads by shifting generation in corrective mode. We believe that in such a case the classical CSCOPF approach is again very risky, since slightly delaying some coordinated corrective actions may lead to cascading trippings of the overloaded branches.

Fig. 1 displays the objective value of our CSCOPF approach, taking as base the objective of the classical CSCOPF, for different increasing values of the relaxation factor $p$. The quite linear shape of this curve is due to the fact that we have used linear (and quite close) bidding prices for all generators. One can observe that the higher the value of $p$ the less the value of the objective. Obviously, the objective value obtained for $p=1$ [respectively, $p>1.58$ ] corresponds to a classical PSCOPF (respectively, CSCOPF) approach. Also, for $p<1.58$ [respectively, $p>1.58$ ], some inequality constraints of type (5) [respectively, (4)] are binding. Such a curve could yield to the system operator valuable information for trading off the risk of cascading events with the price to pay for reducing this risk.

\section{CONCLUSION}

We have reformulated the CSCOPF problem by imposing constraints ensuring existence and viability of the short-term equilibrium point after a contingency is applied and before corrective controls may take place. When these constraints are binding, our approach can provide safer control strategies than the classical CSCOPF would do. We believe that our proposal is a sound compromise between the classical CSCOPF formulation and a full dynamic approach to security control.

Since the size of the extended CSCOPF problem is almost twice that of the classical CSCOPF problem, further work will aim at developing appropriate relaxation techniques to reduce the computational burden, for example by introducing the additional constraints sequentially and only where they are binding, or by exploiting Benders decomposition [2]. 


\section{REFERENCES}

[1] O. Alsac and B. Stott, "Optimal load flow with steady-state security," IEEE Trans. Power App. Syst., vol. PAS-93, no. 3, pp. 745-751, 1974.

[2] A. J. Monticelli, M. V. P. Pereira, and S. Granville, "Security-constrained optimal power flow with post-contingency corrective rescheduling," IEEE Trans. Power Syst., vol. 2, no. 1, pp. 175-182, Feb. 1987.
[3] Long-Term Dynamics, Phase II CIGRE Task Force 38.02.08, 1995.

[4] F. Capitanescu, M. Glavic, D. Ernst, and L. Wehenkel, "Interior-point based algorithms for the solution of optimal power flow problems," Elect. Power Syst. Res. J., vol. 77, no. 5-6, pp. 508-517, Apr. 2007. 